\title{
Prediction of soil water characteristic curve using artificial neural network: a new approach
}

\author{
Abdul-Kareem Esmat Zainal ${ }^{1, *}$ and Shaimaa Hasan Fadhil ${ }^{2}$ \\ ${ }^{1}$ College of Engineering, Baghdad University, Baghdad, Iraq \\ ${ }^{2}$ College of Engineering, Al-Mustansiriyah University, Baghdad, Iraq
}

\begin{abstract}
Soil-Water Characteristic Curve (SWCC) is an important relationship between matric suction and volumetric water content of soils especially when dealing with unsaturated soil problems, these problems may include seepage, bearing capacity, volume change, etc. where the matric or total suction may have a considerable effect on unsaturated soil properties. Obtaining an accurate SWCC for a soil could be cumbersome and sometimes it is time consuming and needs effort for some soils, either through laboratory tests or through field tests. Accurate prediction of this curve can give more precise expectations in design or analysis that include some unsaturated soil properties, which can save more effort and time. This work will concentrate on proposing a new approach for determining the SWCC using Artificial Neural Network (ANN) depending on some soil properties (air-entry point and residual degree of saturation) through computer software MatLab as a tool for ANN. The new approach is to plot the SWCC curve points instead of obtaining the parameters used in Brooks and Corey (BC) Model (1964), van Genuchten (VG) Model (1980), or Fredlund and Xing (FX) Model (1994). Results showed close agreement in determination of the SWCC by verification of the ANN results with an additional curve sample.
\end{abstract}

\section{Introduction}

The SWCC provides a conceptual relation between the mass (and / or volume) of water in a soil and the energy state described as (usually) matric suction of the water phase. The SWCC has proven to be an interpretive model that utilizes the capillary model to provide an understanding of the distribution of water in the voids. The effects of soil texture, void ratio, and gradation became part of the interpretation of measured laboratory SWCC data (i.e. these soil properties are used as parameters in prediction of SWCC).

The SWCCs have an important role in the determination of unsaturated soil property functions (e.g. shear strength, volume change, etc.). The procedures that have been proposed for unsaturated soil properties are approximate but satisfactory for analyzing unsaturated soil mechanics problems [1]. Neural Networks are widely used in experimental researches that need some soil parameters determination either in field or laboratory, and to describe some complex soil behavior.

\section{Previous work}

Many researchers had been working on using Artificial Neural Network (ANN), Genetic Programming (GP), and Genetic Based Neural Network (GBNN) in predicting the SWCC.
Lee, et al., 2005 Considered the change of void ratio during the SWCC tests. Additionally, a method reasonably predicting the SWCC for Korean weathered granite soils was suggested based on the test results obtained from the experiments conducted in their study. A method to estimate the parameters used in Fredlund and Xing's equation were proposed using an ANN (artificial neural network). The particle size distribution, compacted water content and void ratio were used as input data in the ANN model for predicting the parameters.

Johari, et al., 2006 Used Genetic-Based Neural Network (GBNN) is employed to predict the soil-water characteristic curve of unsaturated soils. A three-layer network has been trained by genetic algorithm and its topology is determined by trial and error. The network has five input neurons, namely, initial void ratio, initial gravimetric water content, logarithm of suction normalized with respect to air pressure, clay fraction and silt content.

Johari, et al., 2011 Investigated Genetic-Based Neural Network (GBNN) and Genetic Programming (GP) in determining the SWCC. These two models have identical set of input parameters. The authors utilized some soil properties as parameters to predict the SWCC, these parameters include void ratio, initial water content, clay fraction, silt content and logarithm of suction normalized with respect to air pressure. Assessment of the results indicates that predictions from GBNN model 
have relatively higher accuracy as compared to GP model.

Johari, and Javadi, 2011, Used a neural network which predicts the soil water characteristic curve of unsaturated soils. Their network has five input neurons, namely, initial void ratio, initial gravimetric water content, logarithm of suction normalized with respect to the atmospheric pressure, clay fraction, and silt content.

Johari, and Nejad, 2015, used Gene Expression Programming (GEP) in their research which was employed as an artificial intelligence method for modeling of the SWCC curve. The principal advantage of the GEP approach is its ability to generate powerful predictive equations without any prior assumption on the possible form of the functional relationship. GEP can operate on large quantities of data in order to capture nonlinear and complex relationships between variables of the system. The selected inputs for modeling are the initial void ratio, initial gravimetric water content, logarithm of suction normalized with respect to atmospheric air pressure, clay content, and silt content. The model output is the gravimetric water content corresponding to the assigned input suction.

Nikhil et al., 2016 Used Fredlund and Xing equation that consists of four fitting parameters. The SWCC (fitting parameters) being a function of pore size distribution and stress state vary across different sites, and thus necessitating the need for setting up a sitespecific database for reasonable landslide hazard predictions under an extreme rainfall condition. Therefore, a SWCC fitting parameter estimation model has been developed via an artificial neural network (ANN) using soil samples collected from eight regions in Korea.

\section{Present work}

In this study, examples of ten SWCC curves were taken from experimental tests already published in literature, eight of these curves were used in the learning process of the ANN to obtain the curve points, and another two SWCC curves were used for verifying the reliability of the ANN. These curves are used just to demonstrate the proposed method; larger sets of curves may be used to obtain more accurate results that cover wider ranges of SWCC.

Depending on the typical SWCC shape as shown in Fig. 1 and 2, some ideas are discussed to express the author's point of view in determining the SWCC parameters required for the ANN.

\subsection{Basic assumptions}

In this work, the main difference from other solutions proposed in literature studying how SWCC were obtained is that the main factors (or parameters) that are considered herein are reduced to only two parameters, viz.:
1. The air-entry point value, where the degree of saturation is $100 \%$ and the value of Matric Suction is considered as the first input data in $\mathrm{kPa}$.

2. The $\mathrm{Sr}$ (residual degree of saturation) point, that is considered with its value of $\mathrm{Sr}$ in $\%$, and $\left(\mathrm{u}_{\mathrm{a}}-\mathrm{u}_{\mathrm{w}}\right)_{\mathrm{r}}$ (Matric Suction value at $\mathrm{Sr}$ in $\mathrm{kPa}$ ).

The four input data values are assumed to be the main parameters that can give a reliable SWCC points. The part of SWCC before air-entry point is considered flat, and the part of SWCC after Sr is also considered flat.

Although the SWCC vary between coarse grained soils Fig. 1 and fine grained soils Fig. 2. Since the coarse grained soils have a well-defined air entry point most of the times and a well-defined residual saturation $\mathrm{Sr}$ point. In fine grained soils, these points are curvy and not welldefined, the curve takes a smoother shape which leads to using extrapolation in the curve during its transient stage to obtain a defined points for air-entry and the residual stage.

In addition, the domain of the Soil Suction values are smaller for most coarse grained soils (about few hundreds $\mathrm{kPa}$ ) and much larger for fine grained soils (up to $1 \times 10^{6} \mathrm{kPa}$ ).

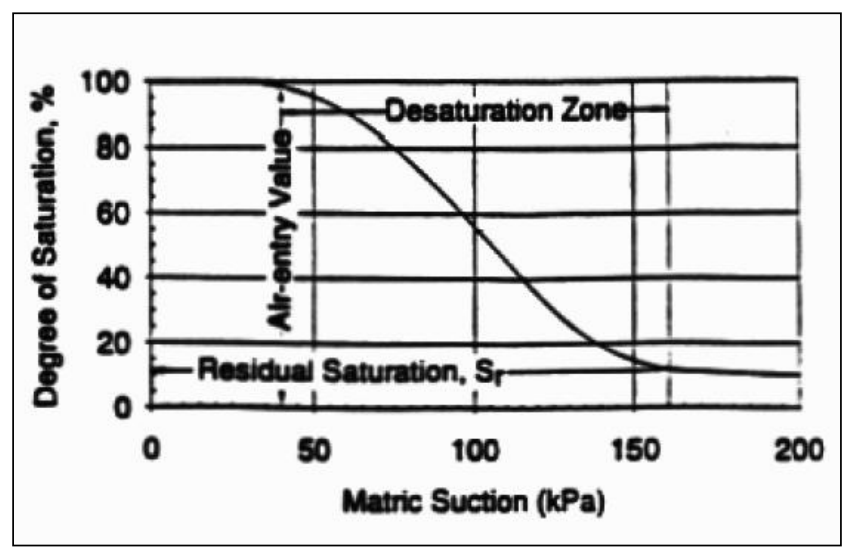

Fig. 1 Typical SWCC for most coarse grained soils [2]

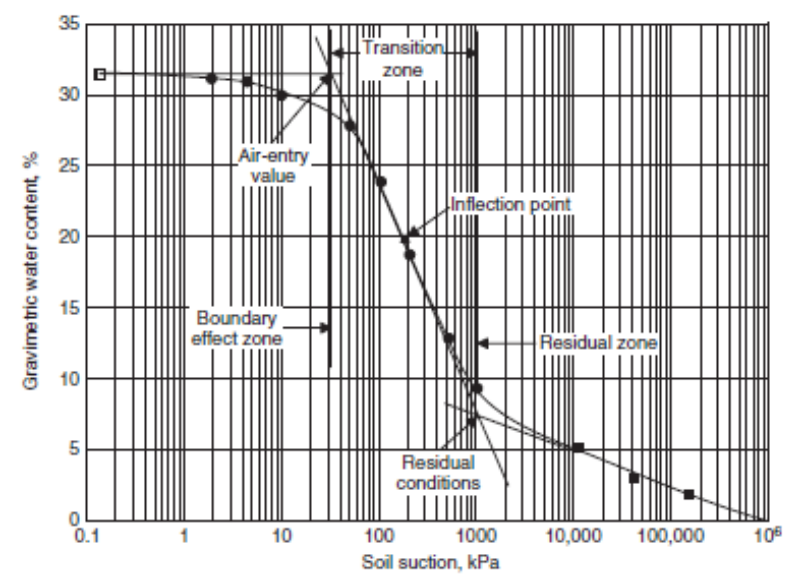

Fig. 2 Typical SWCC for most fine grained soils [1] 
In this work, the air-entry point is considered as it is for coarse grained soils and for fine grained soils, it is taken as the mostly representative point near to $S=100 \%$. For coarse grained soils, the residual degree of saturation point Sr determined as the point with almost flat part at the end of the curve is considered. Where for fine grained soils, the most end flat point at the curve is considered, the purpose of this consideration is to utilize the ANN to provide the shape of the curve as it is, and the user then can define the needed points by using extrapolation.

\subsection{Methodology}

Ten samples of experimentally obtained SWCC were considered in this study, eight of them were used for the learning process, and the other two SWCC were used to verify the reliability of the ANN.

For each curve, the main points of the air-entry point and the $\mathrm{Sr}$ point is defined by their degree of saturation (S) in percent, and the Matric Suction $\left(\mathrm{u}_{\mathrm{a}}-\mathrm{u}_{\mathrm{w}}\right)$ in $\mathrm{kPa}$.

For each SWCC, the curved part between these two points (including these main points) is digitized into 21 equally spaced points (as possible) in the horizontal domain, and the values of the matric suction and the degree of saturation is obtained via a digitizing computer program (Obtaining more points give more accurate results but take more time in digitization). The two main points mentioned (with their four readings) are used as the input data to the Neural Network.

Fig. 3 shows a typical ANN where the input layer, the hidden layer(s) and the output layer are shown in sequence (A, B, C, and D symbols represent Artificial Neurons). Two networks were used to determine the SWCC. The first network has an input layer with four input neurons each represents one input value as follows:

i- $\quad$ Air-entry degree of saturation (in \%),

ii- $\quad$ Air-entry Matric Suction (in $\mathrm{kPa}$ ),

iii- $\quad$ Residual degree of saturation (in \%), and

iv- Residual Matric Suction (in $\mathrm{kPa}$ ).

The hidden layer will contain 20 neurons using logsig function, and the output layer will contain 21 neurons that represent the degree of saturation using purelin function.

The second network has the same input layer, this time it feeds another hidden layer with 20 neurons with logsig function, and connected to another output layer that represents the Matric Suction corresponding to the degrees of saturation obtained from the first network with 21 neurons using purelin function.

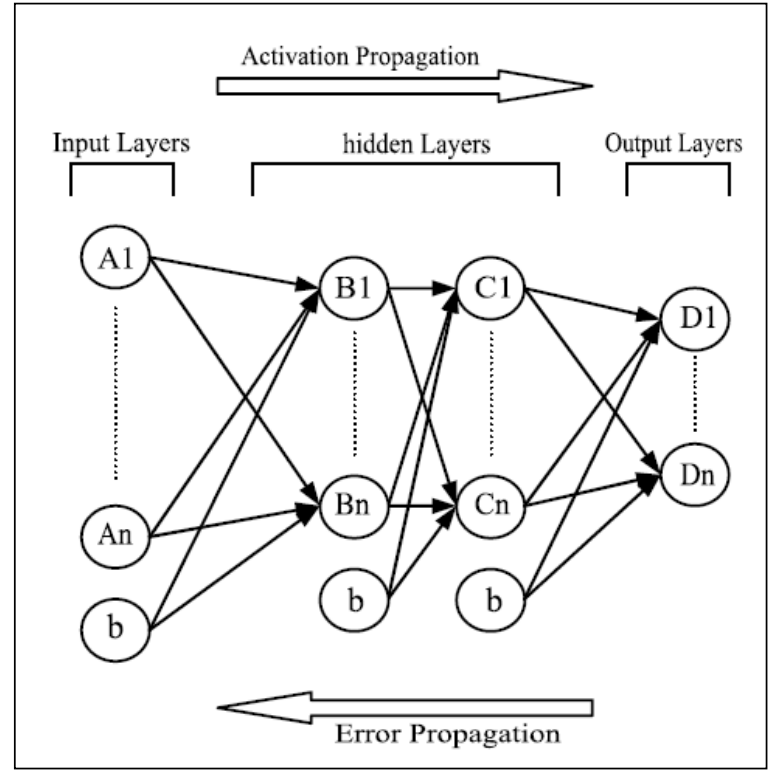

Fig. 3 Typical ANN [4]

\subsection{Working procedure}

The ten SWCC are shown in Fig. 4 to Fig. 9, with the reference of each one, curve 8 was taken in the training process while curves 9 and 10 were taken for the verification process.

All curves were digitized and the data obtained from digitizing these curves are illustrated in Table 1 and Table 2. Table 1 shows the values of the matric suction $\left(\mathrm{u}_{\mathrm{a}}-\mathrm{u}_{\mathrm{w}}\right)$ in $\mathrm{kPa}$ for each curve, where Table 2 shows the corresponding values of the degree of saturation against each value of matric suction. The data are shown in this form as a requirement for the computer software to represent the target values. Table 3 shows the input main points for the curves (air-entry point, and the Sr point).

After the training process was complete, the two ANN (one to represent the matric suction, and the other to represent the degree of saturation) can simulate the verification data.

Results of regression for the matric suction ANN and the degree of saturation ANN are shown in Fig. 10 and Fig. 11 respectively.

The main parameters (four data points) for each curve (namely curve 9 and curve 10) was supplied to each ANN, the input data are shown in Table 4, and the results obtained are shown in Table 5.

Three curves were drawn to represent the results, the first two are the results of verifying data against the digitized data, and the third one is to represent one of the curves already used in the training process. These graphs are shown in Fig. 12, Fig. 13, and Fig. 14. 


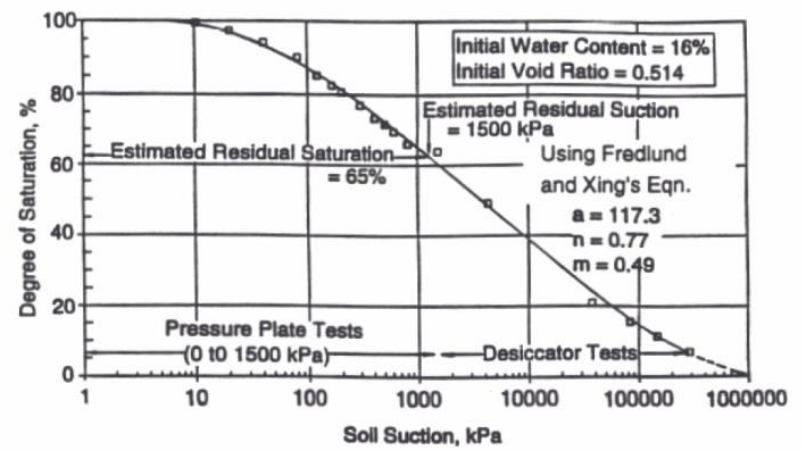

Fig. 4 SWCC for curve 1 after [2]

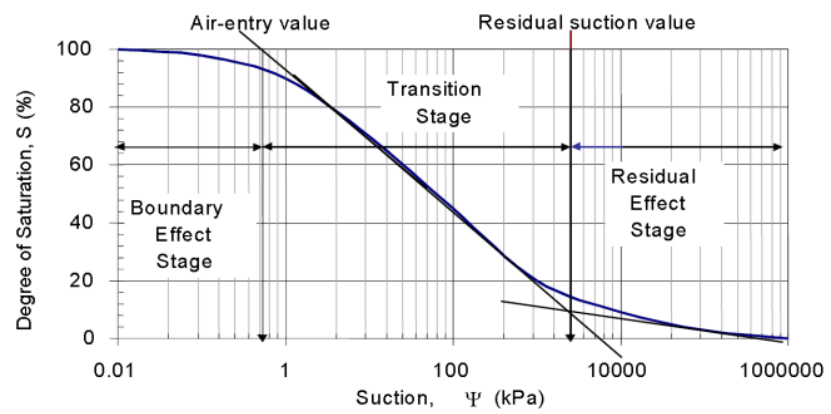

Fig. 5 SWCC for curve 2 after [13]

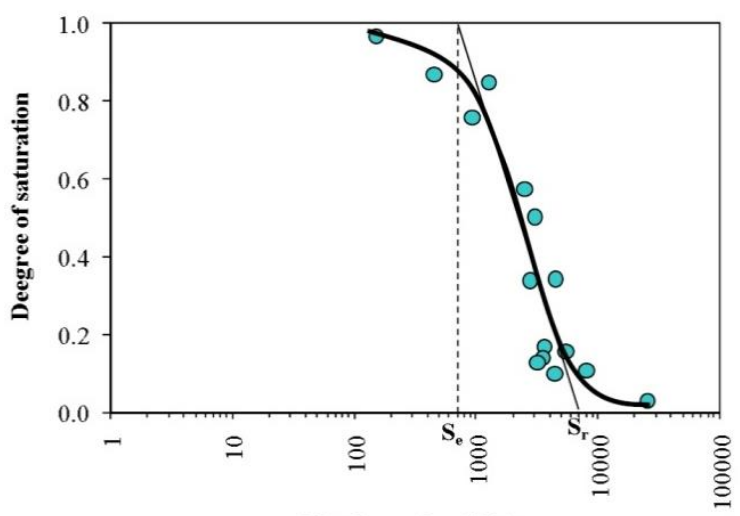

Matric suction (kPa)

Fig. 6 SWCC for curve 3 after [11]

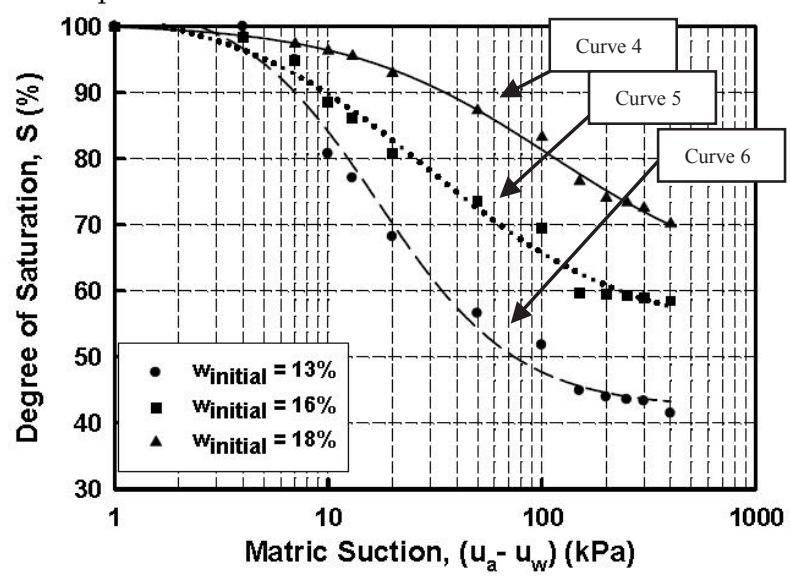

Fig. 7 SWCC for curve 4, 5, and 6 after [12]

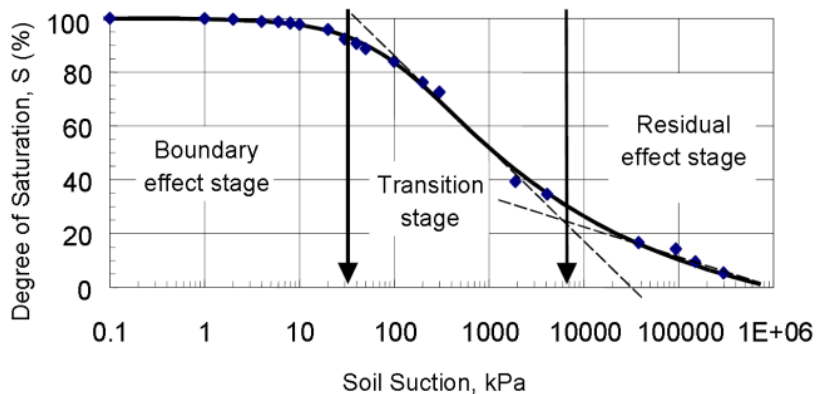

Fig. 8 SWCC for curve 7after [13]

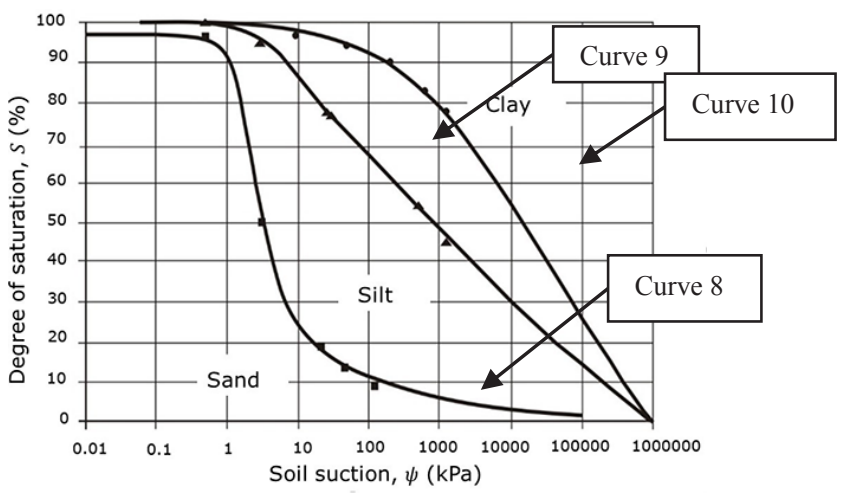

Fig. 9 SWCC for curve 8after [10]

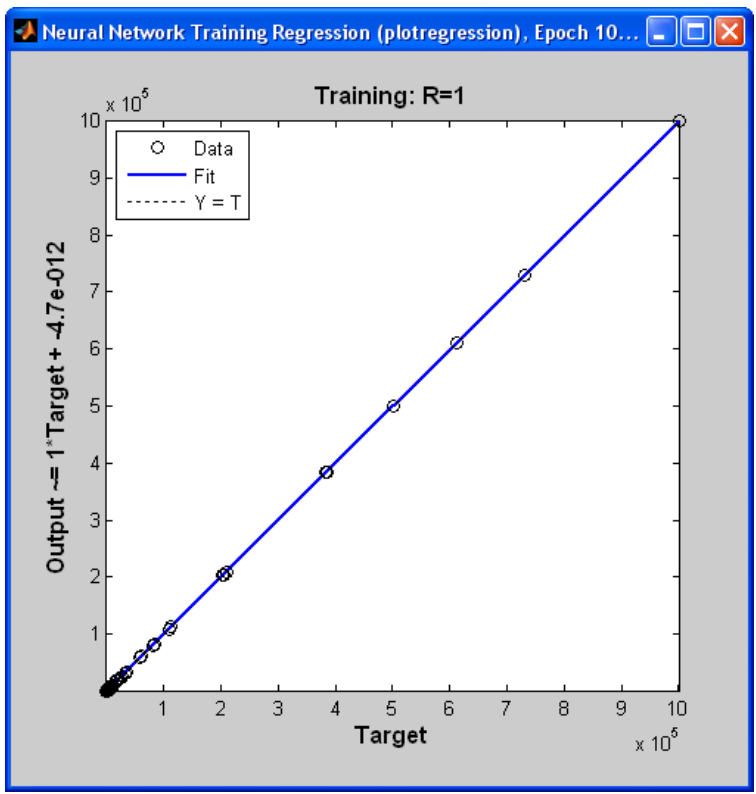

Fig. 10 the Matric Suction Regression result 


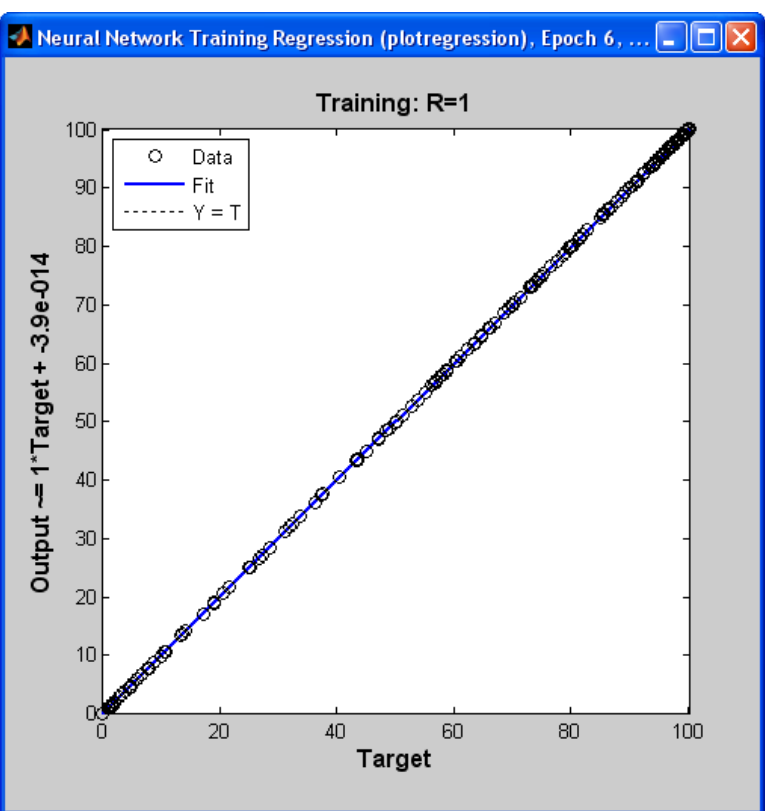

Fig. 11 The degree of saturation regression result

\section{Discussion}

The original curves were obtained from experimental data, the curved lines were digitized to represent the best fit for these experimental points. Results obtained and shown in Fig. 12 to Fig. 14 deviate slightly from the original curved data (as in experimental data points). The best fit lines shown are more representative in showing the curve variation.

Curve 8 best fit line can be represented by (1): with $\mathrm{R}^{2}=0.837$

$$
S=74.14 \Psi^{-(0.079+0.03536 \Psi)}
$$

Also curve 9 best fit line can be represented by (2):

with $\mathrm{R}^{2}=0.9613$

$$
\mathrm{S}=95.12 \Psi^{(0.07386-0.02383 \operatorname{Ln}(\Psi))}
$$

Finally, curve 10 best fit line can be represented by (3):

with $\mathrm{R}^{2}=0.9736$

$$
S=104.5 \Psi\left(-0.01816 \Psi^{0.2011}\right)
$$

where:

$\Psi=$ matric suction $(\mathrm{kPa})$, and

$\mathrm{S}=$ Degree of Saturation in percent.

The trend of these result points is the same as in the original data. It is recommended to use more curves and more data points digitized which can give more accurate curves.

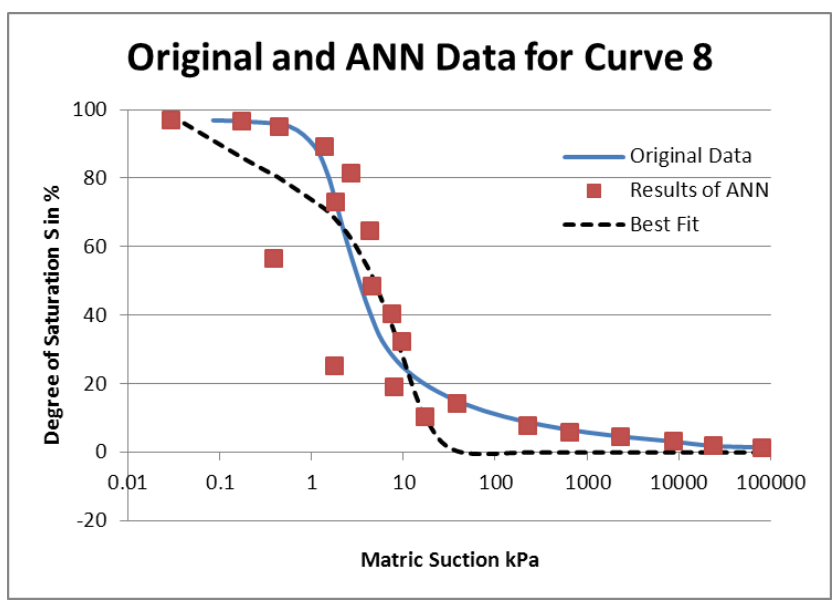

Fig. 12 Original and ANN Data for Curve 8

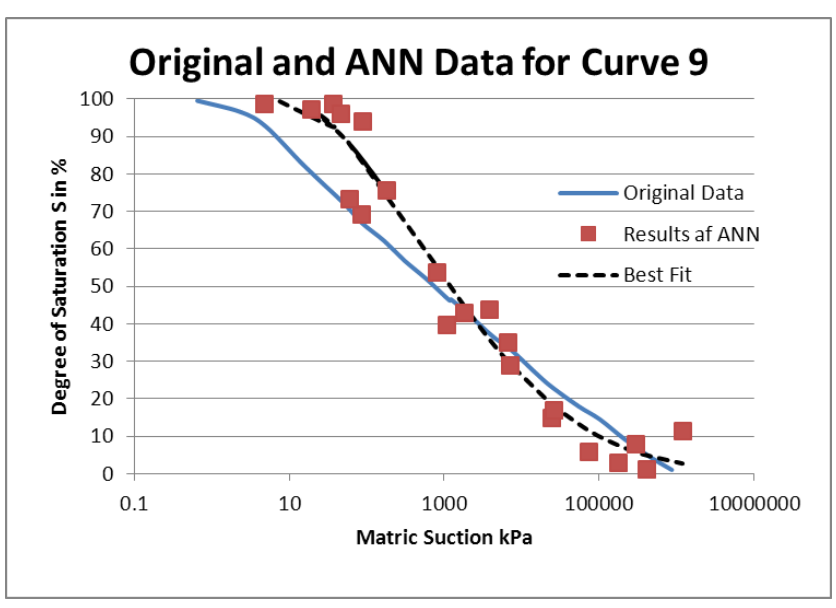

Fig. 13 Original and ANN Data for Curve 9

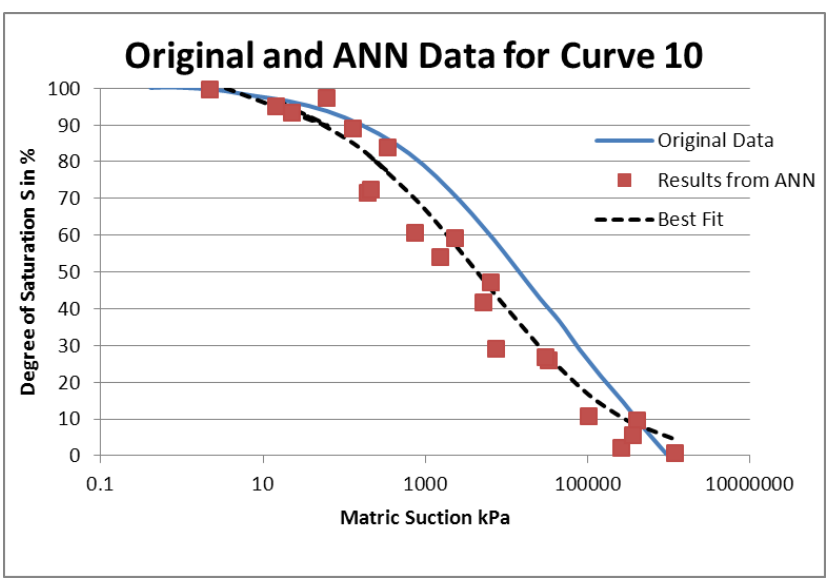

Fig. 14 Original and ANN Data for Curve 10

\section{Conclusions}

The new proposed method of representing the SWCC is found to be satisfactory in representing the SWCC curved segment. Further work in needed to represent the SWCC more accurately and more sample curves are needed.

Other soil properties may be included along with more representatives (i.e. void ratio, soil classification, effective diameter D10, and others). 


\section{References}

[1] D. G. Fredlund, H. Rahardjo, and M. D. Fredlund, "Unsaturated Soil Mechanics in Engineering Practice", John Wiley \& Sons, Inc. (2012).

[2] D. G. Fredlund, S. K.Vanapalli, D. E. Pufahl, "Predicting the shear strength function for unsaturated soils using the soil-water characteristic curve", Unsaturated soils : proceedings of the first International Conference on Unsaturated Soils, UNSAT '95, Paris, France, 6-8 September. Vol. 3. (1995).

[3] A. Johari, A. Hooshmand Nejad, , "Prediction Of Soil-Water Characteristic Curve Using Gene Expression Programming", IJST, Transactions of Civil Engineering, Vol. 39, No. C1, pp. 143-165. (2015)

[4] A. Johari, A. A. Javadi, "Prediction of soil-water characteristic curve using neural network", Unsaturated Soils - Alonso \& Gens (eds) Taylor \& Francis Group, London, pp. 461-466. (2011).

[5] A. Johari, G. Habibagahi, A. Ghahramani, "Prediction of a Soil-Water Characteristic Curve Using a Genetic-Based Neural Network", Scientia Iranica, Vol. 13, No. 3, pp 284-294. (2006).

[6] A. Johari, G. Habibagahi, A. Ghahramani, "Prediction of SWCC using artificial intelligent systems: A comparative study", Scientia Iranica 8 (5),pp 1002-1008. (2011).

[7] S. R. Lee, Y.K. Kim, S. J. Lee, "A Method to Estimate Soil-Water Characteristic Curve For Weathered Granite Soil", Proceedings of the 16th International Conference on Soil Mechanics and Geotechnical Engineering. (2005).

[8] N.V. Nikhil, Yoon Seok, Seung-Rae Lee, DeukHwan Lee, "ANN Based Estimation of SWCC Fitting Parameters for Korean Weathered Soil Considering In-Situ Characteristics", The 2016 world congress on advances on Civil, Environmental, and Materials Research. (ACEM16). (2016).

[9] Won Taek Oh, Sai K. Vanapalli, "Modelling the Stress versus Settlement Behavior of Model Footings in Saturated and Unsaturated Sandy Soils", The 12th International Conference of International Association for Computer Methods and Advances in Geomechanics (IACMAG) 1-6 October, Goa, India.( 2008).

[10] N. Pérez-García, "Determination of Soil-Water Characteristic Curve with pressure plate test". in Groundwater - Contaminant and Resource Management book, Safandila, Querétaro, México: Secretaria de Comunicaciones y Transportes, Mexican Transportation Institute; 54 p. Publicación Técnica No. 313 (in Spanish). (2008)

[11] Anuchit Uchaipichat, "Variation of Pile Capacity in Unsaturated Clay Layer with Suction", Ejge, Vol. 17, pp. 2425-2433. (2012).

[12] S. K, Vanapalli, Taylan Z.N, "Design of Single Piles Using the Mechanics of Unsaturated Soils". Int. Jour. of GEOMATE, March, Vol. (2), No. 1 (Si. No. 3), pp. 197-204.(2012).

[13] S. K., Vanapalli, A. Wright, D.G. Fredlund, " "Shear Strength Behavior of a Silty Soil Over the Suction Range From 0 to $1,000,000 \mathrm{kPa}$ ", 53th Canadian Geotechnical Conference, at Moterial, Quebec,Canada, October. (2000).
Table 1. Matric Suction For the 8 CURVES

\begin{tabular}{|c|c|c|c|c|c|c|c|}
\hline 1 & 2 & 3 & 4 & 5 & 6 & 7 & 8 \\
\hline 8.57553 & 0.055602 & 48.1437 & 1.672545 & 1.694723 & 2.120678 & 1.434355 & 0.084768 \\
\hline 21.06522 & 0.134151 & 137.1151 & 2.653551 & 2.235451 & 2.688841 & 3.182805 & 0.197618 \\
\hline 32.65756 & 0.324312 & 223.0924 & 3.320424 & 2.987829 & 3.593593 & 7.78326 & 0.572914 \\
\hline 62.53066 & 0.756323 & 361.177 & 4.617148 & 4.154408 & 5.198023 & 14.65592 & 1.07374 \\
\hline 117.8381 & 1.671164 & 561.8744 & 5.6271 & 5.700876 & 6.541884 & 27.77551 & 1.485428 \\
\hline 215.9374 & 3.51968 & 809.7844 & 7.134633 & 7.227838 & 8.897422 & 163.9119 & 1.838764 \\
\hline 388.6442 & 7.222818 & 1068.71 & 9.410904 & 9.533255 & 10.73145 & 263.0179 & 2.228015 \\
\hline 689.7889 & 14.55798 & 1319.747 & 12.25082 & 12.40947 & 12.36917 & 428.4782 & 2.746242 \\
\hline 1212.124 & 28.93492 & 1573.873 & 18.62726 & 16.15307 & 15.63406 & 702.5664 & 3.428708 \\
\hline 2117.359 & 56.71134 & 1846.009 & 23.68593 & 22.45854 & 20.86181 & 1151.982 & 4.373265 \\
\hline 3683.958 & 110.2674 & 2143.724 & 28.52934 & 27.73189 & 26.29097 & 1980.802 & 5.922081 \\
\hline 6422.307 & 218.2891 & 2472.965 & 36.11855 & 34.24346 & 32.4627 & 3262.017 & 9.679343 \\
\hline 11218.4 & 421.8968 & 2838.589 & 42.37131 & 42.84465 & 40.79334 & 5645.563 & 19.59148 \\
\hline 19596.84 & 1129.149 & 3269.114 & 51.56069 & 55.03947 & 46.95129 & 9898.265 & 46.6512 \\
\hline 34436.92 & 2545.303 & 3783.747 & 62.65208 & 66.1953 & 56.54949 & 17543.03 & 123.0946 \\
\hline 61363.22 & 5959.629 & 4445.367 & 91.29107 & 97.02138 & 80.60604 & 31771.46 & 343.3754 \\
\hline 112443.7 & 14207.29 & 5489.569 & 132.4373 & 138.5041 & 107.5743 & 58543.65 & 978.5758 \\
\hline 209809.3 & 34140.99 & 7415.32 & 194.6825 & 197.7232 & 164.0409 & 109047.6 & 2812.824 \\
\hline 383060 & 83032.35 & 11011.51 & 254.1643 & 293.6675 & 253.8486 & 204000.5 & 8154.716 \\
\hline 611672.6 & 203560.6 & 17446.28 & 313.848 & 387.3668 & 317.6452 & 384117 & 23742.62 \\
\hline 1000000 & 501059.7 & 26079.9 & 403.2 & 478.3629 & 397.4737 & 729544.6 & 80976.37 \\
\hline & & & & & & &
\end{tabular}

TABLE 2. DegreE OF SATURATION IN \% FOR EACH CURVE

\begin{tabular}{|c|c|c|c|c|c|c|c|}
\hline 1 & 2 & 3 & 4 & 5 & 6 & 7 & 8 \\
\hline 100.1479 & 99.16128 & 100 & 100 & 99.66008 & 100.0255 & 99.60786 & 96.87191 \\
\hline 96.74303 & 97.34698 & 97.73097 & 99.09568 & 98.91423 & 99.65047 & 99.54323 & 96.50971 \\
\hline 94.94827 & 95.10542 & 95.78695 & 98.90598 & 97.98307 & 97.23914 & 97.82207 & 95.09384 \\
\hline 90.41395 & 91.45666 & 93.39106 & 98.34405 & 95.94096 & 93.71603 & 96.99501 & 89.29865 \\
\hline 85.64171 & 86.30029 & 90.02373 & 98.15491 & 94.26917 & 90.93591 & 94.3787 & 81.36319 \\
\hline 80.2874 & 79.98811 & 85.39129 & 97.59489 & 92.59903 & 86.36563 & 77.48374 & 73.0326 \\
\hline 74.53619 & 73.14829 & 79.71971 & 96.47899 & 90.00295 & 82.78463 & 70.42706 & 64.73494 \\
\hline 6.54696 & 66.03213 & 73.41563 & 95.46616 & 87.7772 & 80.08857 & 63.60235 & 56.56898 \\
\hline 62.37258 & 58.71499 & 66.90828 & 93.95669 & 84.99637 & 74.96475 & 56.87706 & 48.46888 \\
\hline 56.14542 & 51.22199 & 60.31059 & 92.38928 & 81.47409 & 69.96314 & 50.11863 & 40.40171 \\
\hline 49.86542 & 43.60339 & 53.69032 & 91.19304 & 79.06441 & 64.6338 & 43.2609 & 32.4004 \\
\hline 43.58533 & 36.16061 & 47.07007 & 89.9752 & 76.65473 & 61.11399 & 37.62905 & 25.12348 \\
\hline 37.33166 & 28.46663 & 40.42723 & 88.53282 & 73.87473 & 57.03875 & 31.99731 & 18.96608 \\
\hline 31.13102 & 19.06192 & 33.78439 & 86.82241 & 71.2792 & 55.04169 & 26.59754 & 14.12578 \\
\hline 24.98315 & 14.18188 & 27.14154 & 85.46153 & 69.42514 & 52.73532 & 21.56227 & 10.50379 \\
\hline 19.02026 & 10.58337 & 20.52125 & 82.0205 & 66.08678 & 48.86303 & 17.15659 & 7.869608 \\
\hline 13.61293 & 7.638194 & 14.17199 & 78.49733 & 63.30403 & 47.19182 & 13.34738 & 5.893974 \\
\hline 8.761576 & 5.069951 & 8.748892 & 75.34393 & 60.52129 & 44.98333 & 9.869533 & 4.445176 \\
\hline 3.805091 & 2.727831 & 4.681239 & 73.28257 & 58.84785 & 43.59684 & 6.689906 & 3.259796 \\
\hline 1.982576 & 1.039097 & 1.923897 & 71.24294 & 58.10199 & 43.48938 & 3.808504 & 1.909779 \\
\hline 0 & 1.009049 & 1.674576 & 69.94256 & 57.72755 & 43.29969 & 1.324733 & 1.448798 \\
\hline & & & & & & &
\end{tabular}

TABLE 3. InPUT DATA

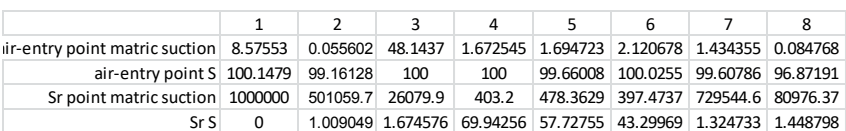
\begin{tabular}{cc|cccccccc} 
SrS & 0 & 1.009049 & 1.674576 & 69.94256 & 57.72755 & 43.29969 & 1.324733 & 1.448798
\end{tabular}

TABle 4. Verifying Data 


\begin{tabular}{|r|c|c|}
\hline & 9 & 10 \\
\hline air-entry point matric suction & 0.651432 & 0.410554 \\
\hline air-entry point S & 99.53902 & 100.1317 \\
\hline Sr point matric suction & 868462.8 & 950007.8 \\
\hline Sr S & 0.987817 & 0.329272 \\
\hline
\end{tabular}

Table 5. Verifying Data Results

\begin{tabular}{|c|c|c|c|}
\hline Matric Suction $\mathrm{kPa}$ & \multicolumn{3}{|c|}{ Degree of Saturation S\% } \\
\hline 9 & 10 & 9 & 10 \\
\hline 4.728393 & 2.223661 & 98.71132 & 99.64459 \\
\hline 36.89925 & 60.00188 & 98.6911 & 97.28264 \\
\hline 19.64693 & 14.42446 & 97.28464 & 95.17319 \\
\hline 46.64776 & 22.40805 & 95.88645 & 93.42735 \\
\hline 88.63865 & -129.106 & 93.829 & 88.91717 \\
\hline 179.7074 & 345.2875 & 75.47142 & 83.76707 \\
\hline 86.4868 & 197.1986 & 69.00925 & 71.41278 \\
\hline 60.14028 & 210.1119 & 73.1 & 72.32742 \\
\hline 811.1791 & 752.5994 & 53.5159 & 60.65005 \\
\hline 1075.403 & 1510.868 & 39.50234 & 53.91856 \\
\hline 1853.102 & 2333.282 & 42.78961 & 59.19435 \\
\hline 3840.476 & 5252.347 & 43.77121 & 41.71416 \\
\hline 6600.492 & 6502.615 & 35.08857 & 47.15224 \\
\hline 7228.543 & 7531.47 & 28.70058 & 28.96275 \\
\hline 24685.07 & 33397.71 & 14.77886 & 25.99016 \\
\hline 26587.29 & 30670.66 & 16.9301 & 26.65161 \\
\hline 74943.67 & 102013.3 & 5.839024 & 10.75616 \\
\hline 178921.8 & 260958.9 & 2.814036 & 2.149345 \\
\hline 298830.6 & 407437.3 & 7.694084 & 9.607634 \\
\hline 409398.9 & 366794.4 & 0.961794 & 5.576248 \\
\hline 1190123 & 1190970 & 11.33338 & 0.769058 \\
\hline & & \\
\hline
\end{tabular}

